\title{
Effect of Synthesis Temperature, Nucleation Time, and Postsynthesis Heat Treatment of ZnO Nanoparticles and Its Sensing Properties
}

\author{
Umair Manzoor, ${ }^{1,2}$ Fatima Tuz Zahra, ${ }^{2}$ Sidra Rafique, ${ }^{2}$ \\ Muhammad Tahir Moin, ${ }^{2}$ and Mohammad Mujahid ${ }^{3}$ \\ ${ }^{1}$ Alamoudi Water Chair, King Saud University, P.O. Box 2460, Riyadh, Saudi Arabia \\ ${ }^{2}$ Center for Micro and Nano Devices, Department of Physics, COMSATS Institute of Information Technology, \\ Islamabad 44000, Pakistan \\ ${ }^{3}$ School of Chemical and Materials Engineering (SCME), National University of Science \& Technology, Islamabad 44000, Pakistan \\ Correspondence should be addressed to Umair Manzoor; umanzoor@ksu.edu.sa
}

Received 21 October 2014; Revised 14 December 2014; Accepted 14 December 2014

Academic Editor: Alan K. T. Lau

Copyright (C) 2015 Umair Manzoor et al. This is an open access article distributed under the Creative Commons Attribution License, which permits unrestricted use, distribution, and reproduction in any medium, provided the original work is properly cited.

\begin{abstract}
Control in size, crystallinity, and optical properties of $\mathrm{ZnO}$ nanoparticles (NPs) synthesized via coprecipitate method were investigated. A systematic change in particle size, crystallinity, and optical properties was observed by increasing synthesis temperature from $65^{\circ} \mathrm{C}$ to $75^{\circ} \mathrm{C}$. A detailed study also suggested that smaller nucleation time is better to control the size distribution but the crystallinity will be compromised accordingly. Postannealing of $\mathrm{ZnO} \mathrm{NPs}$ at $400^{\circ} \mathrm{C}$ also improves the crystal quality. Ultraviolet (UV) sensors were successfully synthesized and the results suggested that as-synthesized ZnO NPs can be used as active material for sensor applications.
\end{abstract}

\section{Introduction}

Zinc oxide $(\mathrm{ZnO})$ has been recognized as one of the promising materials for advance applications because of its wide bandgap $(3.37 \mathrm{eV})$ and relatively large exciton binding energy $(60 \mathrm{meV})[1,2]$. Its potential applications are in transparent electronics, ultraviolet (UV) light emitter, surface acoustic wave (SAW) devices, and spin electronics [3-7]. Various methods have been employed to prepare ZnO NPs with small diameters including precipitation of colloids in solution [8], sol-gel methods [9], thermal transport methods [10, 11], pulsed laser deposition (PLD) [12], and metal-organic chemical vapor deposition (MOCVD) [13]. It is well known that preparation of $\mathrm{ZnO}$ via solution-based chemical processing routes provides a promising option with control of particle size, shape, and crystallinity representing some of the key issues in this area [14].

$\mathrm{ZnO}$ is also a potential optical and gas sensor material due to its high sensitivity to toxic and combustible gases, carrier mobility, and good chemical and thermal stability at moderately higher temperatures [14]. Recently $\mathrm{ZnO}$ shows the possibility of developing photodetectors with intrinsic "visible-blindness" and enables room temperature operation. UV sensing mechanism of $\mathrm{ZnO}$ is linked with bandgap and photoexcitation in which size, crystal structure, and defects play crucial role in sensing response [15].

In the present study, we demonstrate the size and crystal defect relationship of $\mathrm{ZnO}$ NPs by changing synthesis temperature, nucleation time, and annealing, using coprecipitate method. UV light was successfully detected at room temperature using $\mathrm{ZnO}$ NPs synthesized at different temperatures.

\section{Experimental Procedure}

Zinc acetate dihydrate (Sigma-Aldrich, $\mathrm{Zn}(\mathrm{Ac})_{2} \cdot 2 \mathrm{H}_{2} \mathrm{O}$, $1.95 \mathrm{~g}$ ) was added into a flask containing $84 \mathrm{~mL}$ of methanol. Small quantity $(0.5 \mathrm{~mL})$ of distilled water was added to adjust the dielectric constant of the liquid (hamaker constant can be 


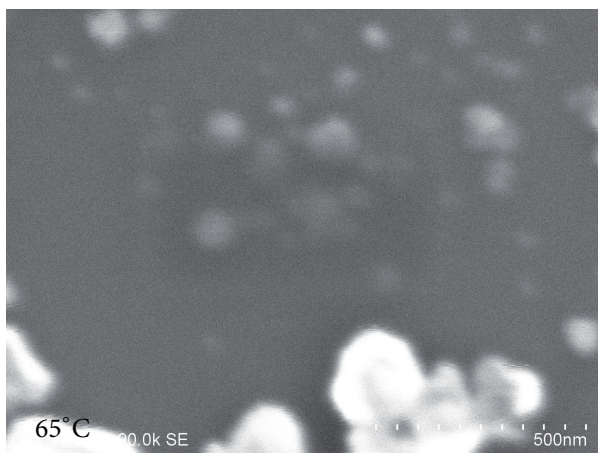

(a)

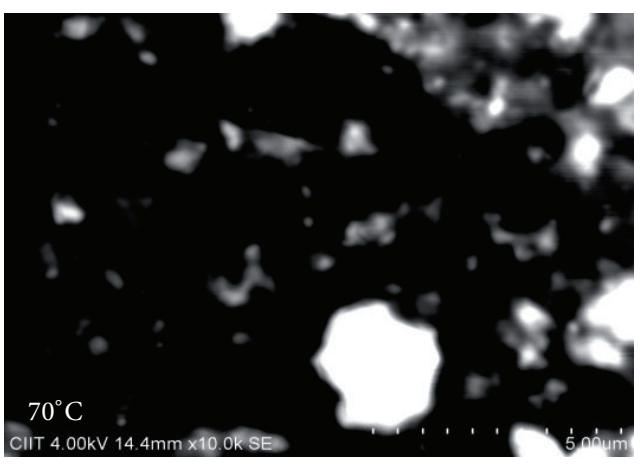

(b)

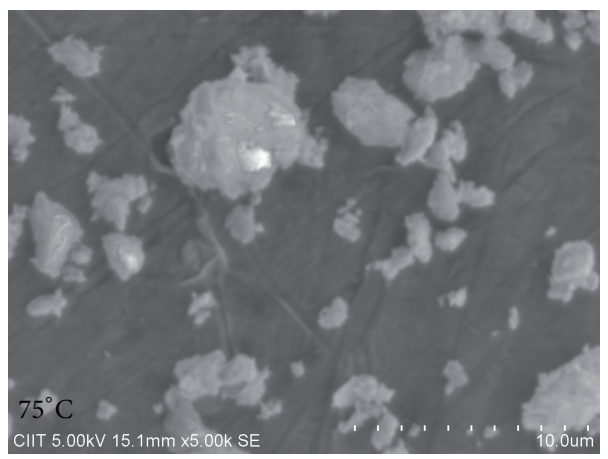

(c)

FIGURE 1: SEM images of ZnO NPs synthesized at (a) $65^{\circ} \mathrm{C}$, (b) $70^{\circ} \mathrm{C}$, and (c) $75^{\circ} \mathrm{C}$. The results clearly suggest increase in particle size with synthesis temperature.

defined for a Van der Waals (VdW) body-body interaction, Wikipedia). The solution was then divided into 3 equal parts and heated to $65^{\circ} \mathrm{C}, 70^{\circ} \mathrm{C}$, and $75^{\circ} \mathrm{C}$. In another beaker, potassium hydroxide $(\mathrm{KOH}, 0.81 \mathrm{~g}$,) was dissolved into $46 \mathrm{~mL}$ of methanol (14.44 mmol, stock solution). Stock solution was then added dropwise to the flask containing $\mathrm{Zn}$ acetate and methanol in approximately 15 minutes. The stock solution and acetate solution were at the same temperatures. The solution was then stirred at a constant temperature of $65^{\circ} \mathrm{C}, 70^{\circ} \mathrm{C}$, and $75^{\circ} \mathrm{C}$ for 2.5 hours. When $\mathrm{KOH}$ was added, the solution became milky and turned transparent after 10 minutes of stirring. After about 1.3 hours of stirring the color of the solution again became milky and remained so till the end. The final solution was then centrifuged to separate $\mathrm{ZnO}$ nanoparticles (NPs) from other solvents. ZnO NPs were washed twice with distilled water and then twice with ethanol and subsequently dried in the oven at $60^{\circ} \mathrm{C}$ for 8 hours.

In another set of experiments, the synthesis temperature was kept constant at $62^{\circ} \mathrm{C}$ and the nucleation time was controlled to $0 \mathrm{~min}, 2 \mathrm{~min}$, and $8 \mathrm{~min}$, to see the effect of nucleation. Heat treatment of selected samples was done at $400^{\circ} \mathrm{C}$ for 2 hours in a tube furnace in air.

The size and shape of the NPs were determined using scanning electron microscope (SEM, Hitachi SU-1500) and atomic force microscope (SPM5200, JEOL) in noncontact (NC) and tapping (AC) modes. The microfabricated cantilever (NSC35; $\mu$ masch) probe employed for this purpose was $130 \mu \mathrm{m}$ long with spring constant, resonance frequency, and nominal tip radius of $4.5 \mathrm{~N} / \mathrm{m}, 150 \mathrm{kHz}$, and $<10 \mathrm{~nm}$, respectively. For AFM sample preparation, $\mathrm{ZnO}$ nanoparticles were dispersed onto atomically smooth highly oriented pyrolytic graphite (HOPG) surface by placing a droplet on it from the suspension and drying off the liquid medium. Phase analysis was done using X-ray diffraction (XRD, PANalytical $\mathrm{X}$-Pert Pro with $\mathrm{Cu}-\mathrm{K} \alpha$ radiations). Optical characterization was carried out by measuring the diffuse reflectance spectroscopy (DRS) at room temperature using Lemda-950 Perkin-Elmer and Fourier transform infrared spectrometer (FTIR). UV sensor experiments were done using $18 \mathrm{~W} \mathrm{UV}$ lamp.

\section{Results and Discussion}

Figure 1 shows the SEM micrographs of ZnO NPs after washing and drying. SEM results show an increase in the average particle size and particle agglomeration for higher synthesis temperatures. The particle sizes were estimated to be $98 \pm 43$, $135 \pm 77$, and $458 \pm 243 \mathrm{~nm}$, for synthesis temperatures of $65^{\circ} \mathrm{C}, 70^{\circ} \mathrm{C}$, and $75^{\circ} \mathrm{C}$, respectively. Nanoparticles were found to be nearly spherical in shape.

Figure 2 is the XRD results of all the three samples. The results clearly suggest that there is a systematic decrease in peak intensity and increase in peak width (FWHM) with increase in temperature (inset). Figure 1 clearly shows that particle size is increasing with temperature. Therefore, 


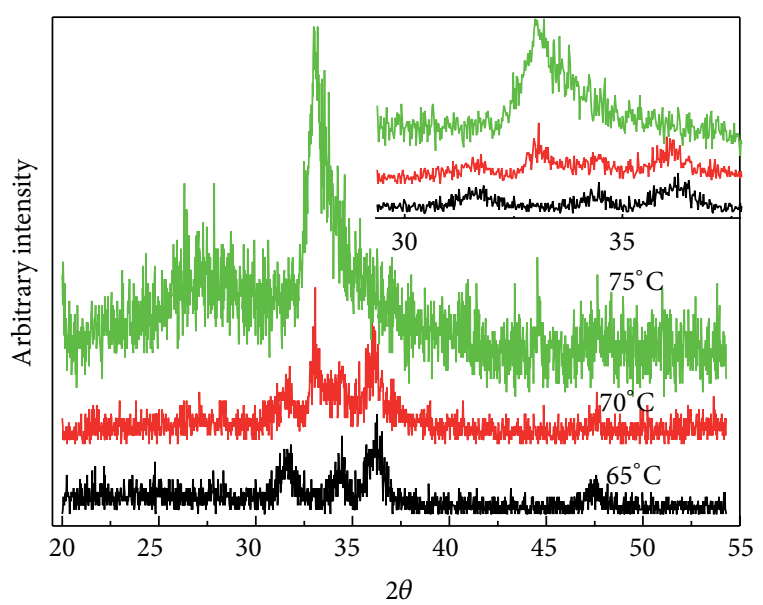

Figure 2: XRD date of ZnO NPs synthesized at $65^{\circ} \mathrm{C}, 70^{\circ} \mathrm{C}$, and $75^{\circ} \mathrm{C}$ shows that crystallinity is better with lower synthesis temperatures. The inset gives a clear indication of peak shift with different synthesis temperatures.

FWHM effect may not be the size or quantum confinement effects but possible reason for increase in FWHM can be that crystal quality was decreasing and defects increased with temperatures. It is suggested that fast growth rate at higher temperatures can be the possible reasons for this phenomenon. This can also be predicted by von Weymarn ratio (commonly misspelt as von Weimarn), where, according to this relation, the particle size is inversely proportional to relative supersaturation where

$$
\text { Relative Supersaturation }=\frac{(Q-S)}{S},
$$

where $Q$ is the concentration of reactants before precipitation, $S$ is the solubility of precipitate in the medium from which it is being precipitated [16]. There is another possibility that this is not $\mathrm{ZnO}$ but a mixture of hydroxides [17]. However, we did not find strong evidence in the XRD data and according to our understanding crystallinity related discussion best explains our results.

Figure 3 shows the FTIR spectrum acquired in the range of $420-4000 \mathrm{~cm}^{-1}$. ZnO stretching mode appeared at $480 \mathrm{~cm}^{-1}$ [18]. A small signal of $(\mathrm{OH})$ groups around $3570 \mathrm{~cm}^{-1}$ is observed probably arising due to contact of the $\mathrm{ZnO}$ sample with air resulting in adsorption of water vapor. Lower intensities of secondary peaks are clear indication that washing of samples was to a reasonable level.

Another set of experiments were performed by systematically changing the nucleation time ( $0 \mathrm{~min}, 2 \mathrm{~min}$, and $8 \mathrm{~min}$ ) while keeping all the other synthesis parameters constant. The area scans for ZnO NPs are presented in Figure 4. The presented selected scan area gives insight into the particle shape and size that is representative of the bulk powder sample. Figure 4 clearly suggests a systematic increase in the average particle size and wider size distribution for higher nucleation times. Particle sizes were estimated to be $41 \pm 11$, $84 \pm 21$, and $135 \pm 95 \mathrm{~nm}$, for nucleation time of $0 \mathrm{~min}$, $2 \mathrm{~min}$, and $8 \mathrm{~min}$, respectively (Table 1 ). Nanoparticles were

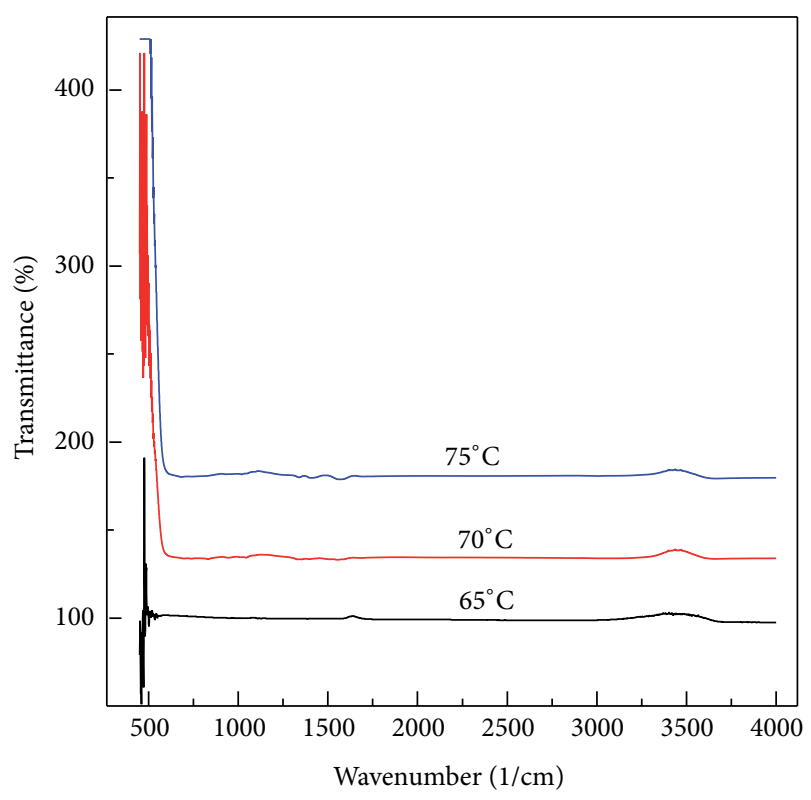

FIGURE 3: FTIR data of all the 3 samples shows $\mathrm{Zn}-\mathrm{O}$ related peaks. The results also suggest that washing of samples was to a reasonable level.

TABLE 1: Effect of nucleation time on size and optical properties of ZnO NPs.

\begin{tabular}{lccc}
\hline $\begin{array}{l}\text { Nucleation time } \\
(\mathrm{min})\end{array}$ & $\begin{array}{c}\text { Particle size } \\
\text { from AFM } \\
\text { images }(\mathrm{nm})\end{array}$ & $\begin{array}{c}\text { Crystallite size } \\
\text { from }(101) \text { peak } \\
(\mathrm{nm})\end{array}$ & $\begin{array}{c}\text { Bandgap } \\
(\mathrm{eV})\end{array}$ \\
\hline 0 & $41 \pm 11$ & 20 & 3.1 \\
2 & $84 \pm 21$ & 24 & 3.1 \\
8 & $135 \pm 95$ & 57 & 3.2 \\
\hline
\end{tabular}

found to be nearly spherical in shape with narrow particle size distribution. A series of experiments (results not shown here) indicated the important role of stirring toward control of size distribution. It was noticed that intermediate stirring offered better control over particle size, producing nanoparticles with relatively narrow size distribution.

Figure 5 is the XRD results of all the three samples with different nucleation time. The results clearly suggest that there is a systematic increase in FWHM and peak shift towards higher angle with increase in nucleation time. The angular peak position of bulk crystalline $\mathrm{ZnO}$ with (101) orientation is $2 \theta=36.255^{\circ}$ (JCPDS card \# 65-3411) [19]. XRD results suggest that, with the increase in nucleation time, the peak shifts towards higher angle which is much closer to the above-mentioned JCPDS card. One of the possible scientific explanations can be that if nucleation sets in too quickly, too many crystals will grow and reduce the local reactants concentration and defects started appearing into the crystal, affecting the crystal quality. However, the size distribution will become wider with the increase in the nucleation time.

The UV-visible spectroscopy results are shown in Figure 6. The diffuse reflectance, $R$, of the samples is 


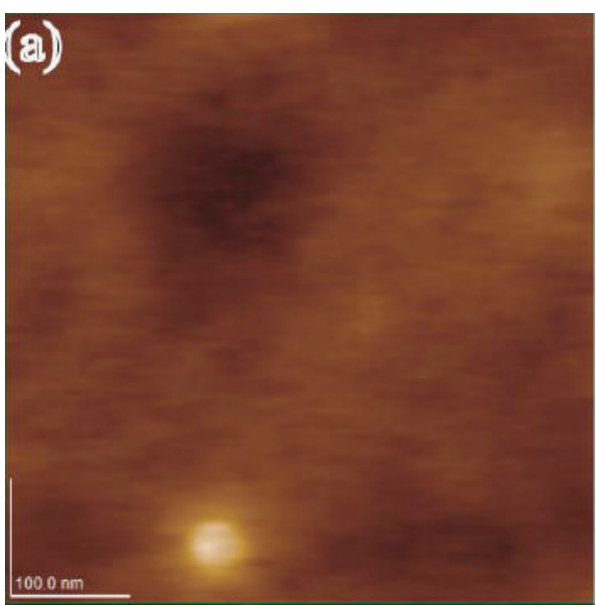

(a)

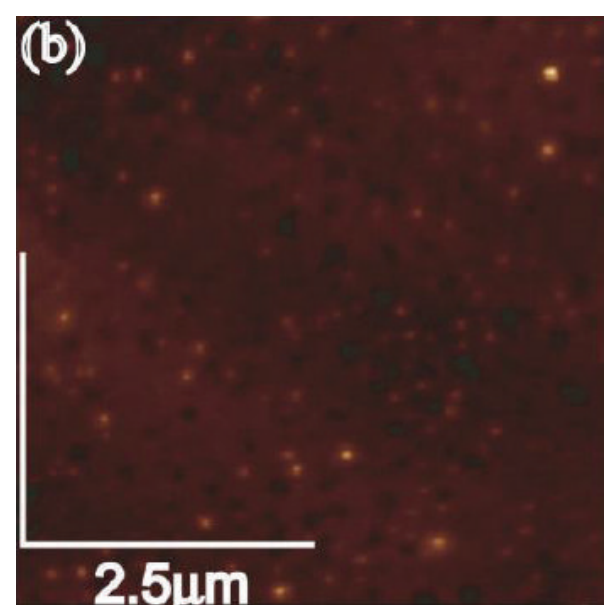

(b)

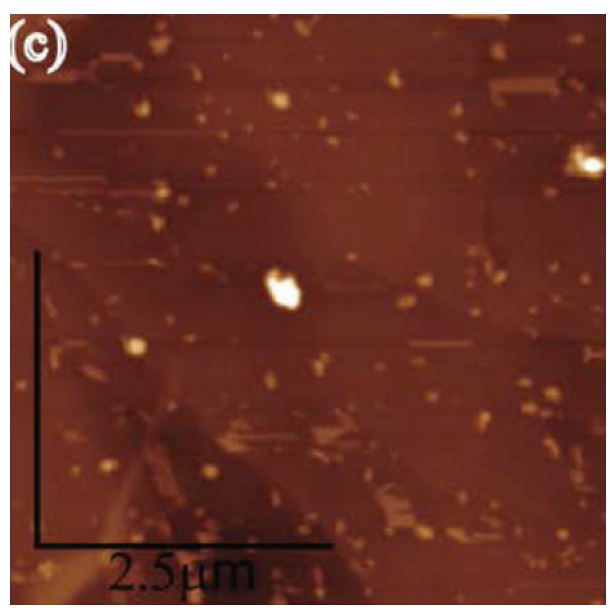

(c)

Figure 4: AFM images of ZnO NPs with nucleation time of (a) $0 \mathrm{~min}$, (b) $2 \mathrm{~min}$, and (c) $8 \mathrm{~min}$. The results show that overall particle size and size distribution increase with increase in nucleation time.

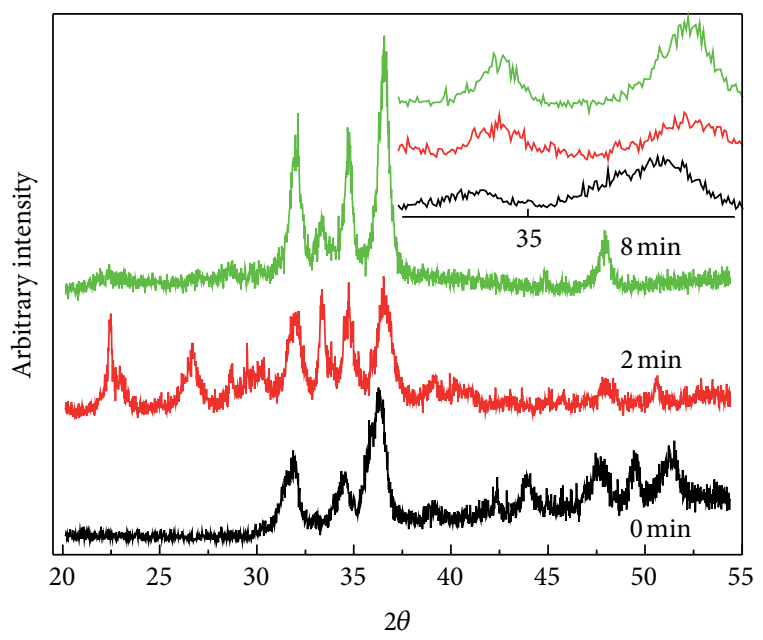

FIGURE 5: XRD peaks of ZnO NPs with nucleation time of (a) 0 min, (b) $2 \mathrm{~min}$, and (c) $8 \mathrm{~min}$. The crystallinity of samples becomes better when nucleation time is increased. related to the Kubelka-Munk function $F(R)$ by the relation $F(R)=(1-R)^{2} / 2 R$, where $R$ is the percentage reflectance [19]. The spectra used for the bandgap calculations are plotted in terms of $F^{2}(R)$. The bandgap energy of the $\mathrm{ZnO}$ NPs was calculated from their diffuse reflectance spectra by plotting the square of the Kubelka-Munk function $F(R)^{2}$ versus energy in electron volts. The linear part of the curve was extrapolated to $F(R)^{2}=0$ to get the direct bandgap energy. There is a slight change in the bandgap with the increase in the nucleation time (Table 1) and the bandgap ranges between $3.0 \mathrm{eV}$ and $3.2 \mathrm{eV}$. This is in accordance with the previously reported results [11]. The results also suggest that the intensity of deep level emissions (DLE, defects related peak, 2.3 2.7 eV) decreases with increase in the nucleation time. Point defects, that is, oxygen vacancy, oxygen interstitial, zinc vacancy, and impurities, are considered to be possible origins for these bands [11]. The decrease in DLE suggests that crystal quality becomes better with increase in nucleation time. 


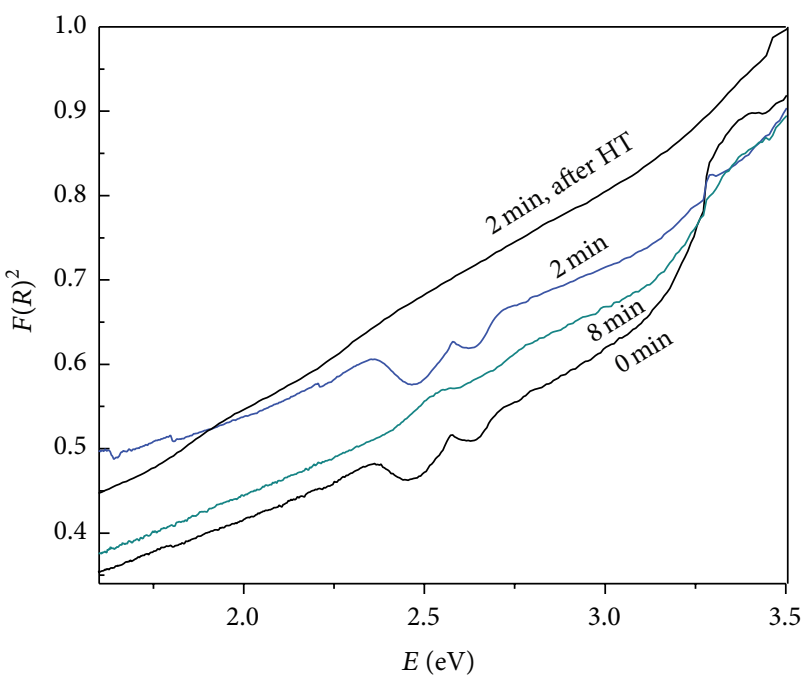

Figure 6: UV-Vis spectroscopy results show direct band emission peak at around $3.2 \mathrm{~nm}$ and defect related DLE peaks in the range of $2.4 \sim 2.7 \mathrm{~nm}$ for $\mathrm{ZnO}$ NPs with nucleation time of $0 \mathrm{~min}, 2 \mathrm{~min}$, and $8 \mathrm{~min}$. The results also show that DLE peaks disappear after annealing the sample (nucleation time: $2 \mathrm{~min}$ ) at $400^{\circ} \mathrm{C}$.

The XRD and UV-Vis results are in excellent agreement with each other. The increase in XRD intensities suggests better quality of $\mathrm{ZnO}$. XRD peak shift towards higher angle also indicates an improvement in the overall crystal structure. Hence it can be suggested that, in coprecipitate method, by increasing the nucleation time, ionic depletion regions around the nuclei can be avoided and supersaturation conditions will not be disturbed. This gives enhanced mobility and diffusion that could decrease the defects and improve crystal quality of ZnO NPs [20].

In another set of experiments, $\mathrm{ZnO}$ NPs were annealed in air at $400^{\circ} \mathrm{C}$ to tune the crystal defects. The results in Figure 6 (nucleation time: $2 \mathrm{~min}$, before and after annealing) clearly suggest that DLE intensity significantly decreases after annealing. Previous researchers have suggested that defects may degrade the performance of optical devices fabricated from III to V semiconductors [20]. Two different groups in independent studies concluded that after annealing the $\mathrm{ZnO}$ films, DLE peak decreases significantly, indicating that quality of $\mathrm{ZnO}$ film was improved through annealing [21]. Therefore it can be deduced that postsynthesis heat treatment plays an important role in tuning the crystal defects. Point defects, that is, oxygen vacancy, oxygen interstitial, zinc vacancy, and impurities, are considered to be possible origins for these bands [22]. Point defects, at compound semiconductor surfaces, are, for entropy reasons, thermodynamically stable at high temperatures [23]. Therefore it is difficult to remove them completely only by thermal treatment and a minor peak may always be present in the UV-Vis data.

Figure 7 is the room temperature UV sensing results of $\mathrm{ZnO}$ NPs. The overall resistance decreases with the exposure of UV light and increases again when the UV lamp was switched off. When the energy of photon is greater than the band gap energy $E_{g}$, radiation is absorbed by $\mathrm{ZnO} \mathrm{NPs}$,
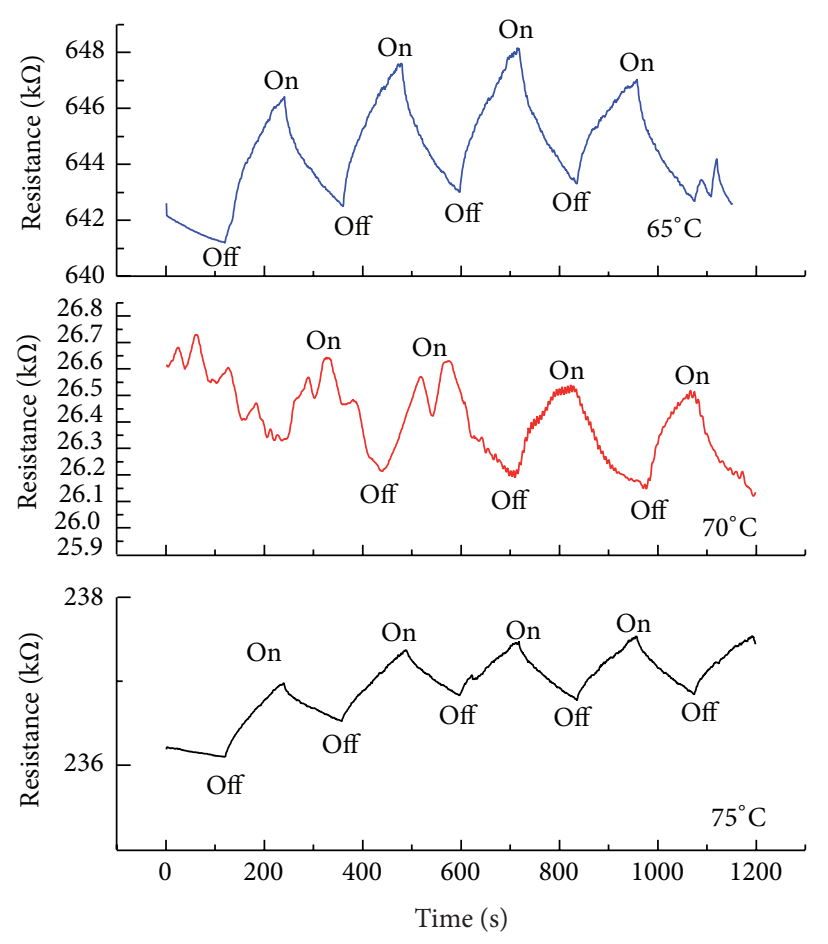

FIGURE 7: UV sensing results of ZnO NPs synthesized at different temperatures. The results give a clear indication that NPs synthesized at $65^{\circ} \mathrm{C}$ show highest sensitivity.

creating electron-hole pairs. The photogenerated, positively charged hole neutralizes the chemisorbed oxygen responsible for the higher resistance, increasing the conductivity of the device. As a consequence, the conductivity in the material increases, giving rise to photocurrent. This results in decrease in overall resistance. This process goes on in a cyclic manner with the On-Off switching of UV light. Particles synthesized at $65^{\circ} \mathrm{C}$ showed best results as UV sensors; that is, sensitivity was highest as compared to NPs synthesized at $70^{\circ} \mathrm{C}$ and $75^{\circ} \mathrm{C}$. ZnO NPs synthesized at $70^{\circ} \mathrm{C}$ showed intermediate sensitivity and least sensitivity was shown by NPs synthesized at $75^{\circ} \mathrm{C}$. This effect can be related to the crystal quality of $\mathrm{ZnO}$ NPs (related to defects and bandgap). If there are lesser crystal defects the DLE emissions will be lesser and more photons will be available to excite the electrons from valance to conduction band, thus increasing the photocurrent. Also, some irregular peaks were observed in the sensor results of NPs synthesized at $70^{\circ} \mathrm{C}$. This may be due to the fluctuations in the light source for that particular experiment.

\section{Conclusion}

$\mathrm{ZnO}$ NPs were synthesized by coprecipitate method. The particle sizes were estimated to be $98 \pm 43,135 \pm 77$, and $458 \pm 243 \mathrm{~nm}$, for synthesis temperatures of $65^{\circ} \mathrm{C}, 70^{\circ} \mathrm{C}$, and $75^{\circ} \mathrm{C}$, respectively. XRD results suggested that faster growth dynamics at higher temperatures introduce defects and therefore decrease the crystal quality. Nucleation time is also critical to control the size and size distribution. Particle 
sizes were $41 \pm 11 \mathrm{~nm}, 84 \pm 21 \mathrm{~nm}$, and $135 \pm 95 \mathrm{~nm}$, for nucleation time of $0 \mathrm{~min}, 2 \mathrm{~min}$, and $8 \mathrm{~min}$, respectively. However, XRD results clearly suggested a decrease in crystallinity with decrease in particle size. Therefore a compromise is always there between smaller size of $\mathrm{ZnO}$ NPs and the crystal defects. UV-Vis results also support the findings and DLE peaks significantly decrease with increase in nucleation time. UV-Vis data of as-synthesized and annealed samples also suggested a significant decrease in the DLE peaks after postsynthesis annealing. Comparison of UV sensors results suggested that best sensitivity was from $\mathrm{ZnO}$ NPs with best crystal quality that is synthesized at $65^{\circ} \mathrm{C}$.

\section{Conflict of Interests}

The authors declare that there is no conflict of interests regarding the publication of this paper.

\section{Acknowledgment}

This project was supported by NSTIP Strategic Technologies Program (no. 12-WAT-2451-02) in the Kingdom of Saudi Arabia.

\section{References}

[1] W. I. Park, G.-C. Yi, M. Y. Kim, and S. J. Pennycook, "Quantum confinement observed in $\mathrm{ZnO} / \mathrm{ZnMgO}$ nanorod heterostructures," Advanced Materials, vol. 15, no. 6, pp. 526-529, 2003.

[2] J.-K. Song, M.-B. Zheng, Z.-J. Yang et al., "Synthesis of novel flower-like $\mathrm{Zn}(\mathrm{OH}) \mathrm{F}$ via a microwave-assisted ionic liquid route and transformation into nanoporous $\mathrm{ZnO}$ by heat treatment," Nanoscale Research Letters, vol. 4, no. 12, pp. 1512-1516, 2009.

[3] H.-Q. Wu, X.-W. Wei, M.-W. Shao, and J.-S. Gu, "Synthesis of zinc oxide nanorods using carbon nanotubes as templates," Journal of Crystal Growth, vol. 265, no. 1-2, pp. 184-189, 2004.

[4] J. Sun, J. Bian, H. Liang et al., "Realization of controllable etching for $\mathrm{ZnO}$ film by $\mathrm{NH} 4 \mathrm{Cl}$ aqueous solution and its influence on optical and electrical properties," Applied Surface Science, vol. 253, no. 11, pp. 5161-5165, 2007.

[5] X. Zhong and W. Knoll, "Morphology-controlled large-scale synthesis of $\mathrm{ZnO}$ nanocrystals from bulk $\mathrm{ZnO}$," Chemical Communications, no. 9, pp. 1158-1160, 2005.

[6] Ü. Özgür, Y. I. Alivov, C. Liu et al., "A comprehensive review of ZnO materials and devices," Journal of Applied Physics, vol. 98, no. 4, Article ID 041301, pp. 1-103, 2005.

[7] X. Zhang, L. Wang, and G. Zhou, "Synthesis of well-aligned $\mathrm{ZnO}$ nanowires without catalysts," Reviews on Advanced Materials Science, vol. 10, no. 1, pp. 69-72, 2005.

[8] U. Koch, A. Fojtik, H. Weller, and A. Henglein, "Photochemistry of semiconductor colloids. Preparation of extremely small $\mathrm{ZnO}$ particles, fluorescence phenomena and size quantization effects," Chemical Physics Letters, vol. 122, no. 5, pp. 507-510, 1985.

[9] C. Cannas, M. Casu, A. Lai, A. Musinu, and G. Piccaluga, "XRD, TEM and ${ }^{29} \mathrm{Si}$ MAS NMR study of sol-gel $\mathrm{ZnO}-\mathrm{SiO}_{2}$ nanocomposites," Journal of Materials Chemistry, vol. 9, no. 8, pp. 1765-1769, 1999.
[10] U. Manzoor, D. K. Kim, M. Islam, and A. S. Bhatti, "Removal of micrometer size morphological defects and enhancement of ultraviolet emission by thermal treatment of Ga-doped $\mathrm{ZnO}$ nanostructures," PLoS ONE, vol. 9, no. 1, Article ID e86418, 2014.

[11] U. Manzoor and D. K. Kim, "Size control of $\mathrm{ZnO}$ nanostructures formed in different temperature zones by varying Ar flow rate with tunable optical properties," Physica E: Low-Dimensional Systems and Nanostructures, vol. 41, no. 3, pp. 500-505, 2009.

[12] I. Ozerov, A. V. Bulgakov, D. K. Nelson, R. Castell, and W. Marine, "Production of gas phase zinc oxide nanoclusters by pulsed laser ablation," Applied Surface Science, vol. 247, no. 1-4, pp. 1-7, 2005.

[13] B. Hahn, G. Heindel, E. Pschorr-Schoberer, and W. Gebhardt, "MOCVD layer growth of $\mathrm{ZnO}$ using DMZn and tertiary butanol," Semiconductor Science and Technology, vol. 13, no. 7, pp. 788-791, 1998.

[14] T. Gao and T. H. Wang, "Synthesis and properties of multipodshaped $\mathrm{ZnO}$ nanorods for gas-sensor applications," Applied Physics A, vol. 80, no. 7, pp. 1451-1454, 2005.

[15] Y. H. Liu, S.-J. Young, C. H. Hsiao et al., "Visible-blind photodetectors with $\mathrm{Mg}$-doped $\mathrm{ZnO}$ nanorods," IEEE Photonics Technology Letters, vol. 26, no. 7, pp. 645-648, 2014.

[16] F. Gao, C. Lv, J. Han et al., "CdTe-montmorillonite nanocomposites: control synthesis, UV radiation-dependent photoluminescence, and enhanced latent fingerprint detection," The Journal of Physical Chemistry C, vol. 115, no. 44, pp. 21574-21583, 2011.

[17] A. S. Shaporev, V. K. Ivanov, A. E. Baranchikov, O. S. Polezhaeva, and Y. D. Tret'yakov, "ZnO formation under hydrothermal conditions from zinc hydroxide compounds with various chemical histories," Russian Journal of Inorganic Chemistry, vol. 52, no. 12, pp. 1811-1816, 2007.

[18] R. Wahab, S. G. Ansari, Y. S. Kim et al., "Low temperature solution synthesis and characterization of $\mathrm{ZnO}$ nano-flowers," Materials Research Bulletin, vol. 42, no. 9, pp. 1640-1648, 2007.

[19] U. Manzoor, M. Islam, L. Tabassam, and S. U. Rahman, "Quantum confinement effect in $\mathrm{ZnO}$ nanoparticles synthesized by co-precipitate method," Physica E: Low-Dimensional Systems and Nanostructures, vol. 41, no. 9, pp. 1669-1672, 2009.

[20] Z. B. Fang, Z. J. Yan, Y. S. Tan, X. Q. Liu, and Y. Y. Wang, "Influence of post-annealing treatment on the structure properties of ZnO films," Applied Surface Science, vol. 241, no. 3-4, pp. 303308, 2005.

[21] B. Lin, Z. Fu, and Y. Jia, "Green luminescent center in undoped zinc oxide films deposited on silicon substrates," Applied Physics Letters, vol. 79, no. 7, article 943, 2001.

[22] K. Vanheusden, W. L. Warren, C. H. Seager, D. R. Tallant, J. A. Voigt, and B. E. Gnade, "Mechanisms behind green photoluminescence in $\mathrm{ZnO}$ phosphor powders," Journal of Applied Physics, vol. 79, no. 10, pp. 7983-7990, 1996.

[23] W. Gopel, "Initial steps of interface formation: surface states and thermodynamics," Journal of Vacuum Science \& Technology, vol. 16, no. 5, pp. 1229-1235, 1979. 

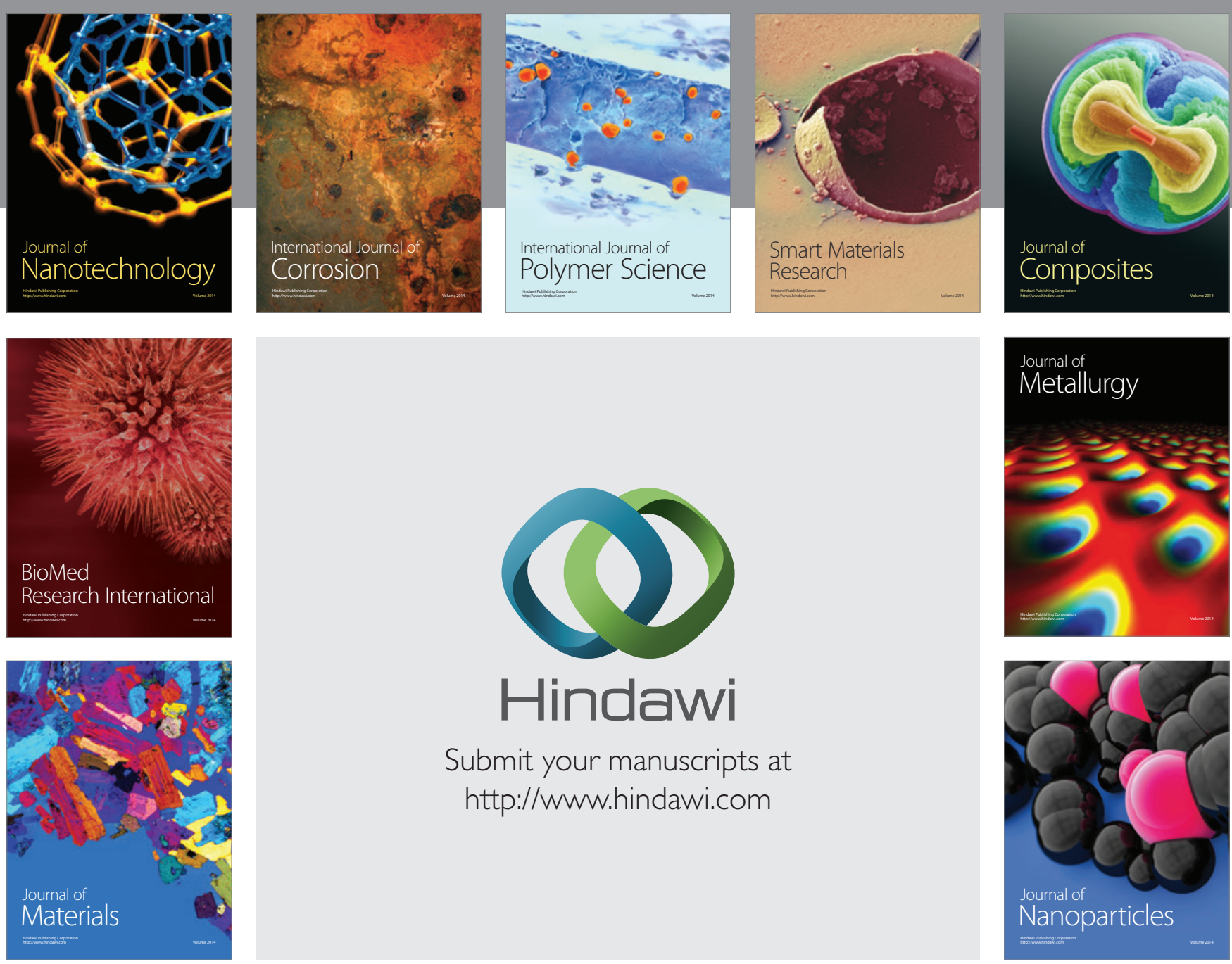

Submit your manuscripts at http://www.hindawi.com
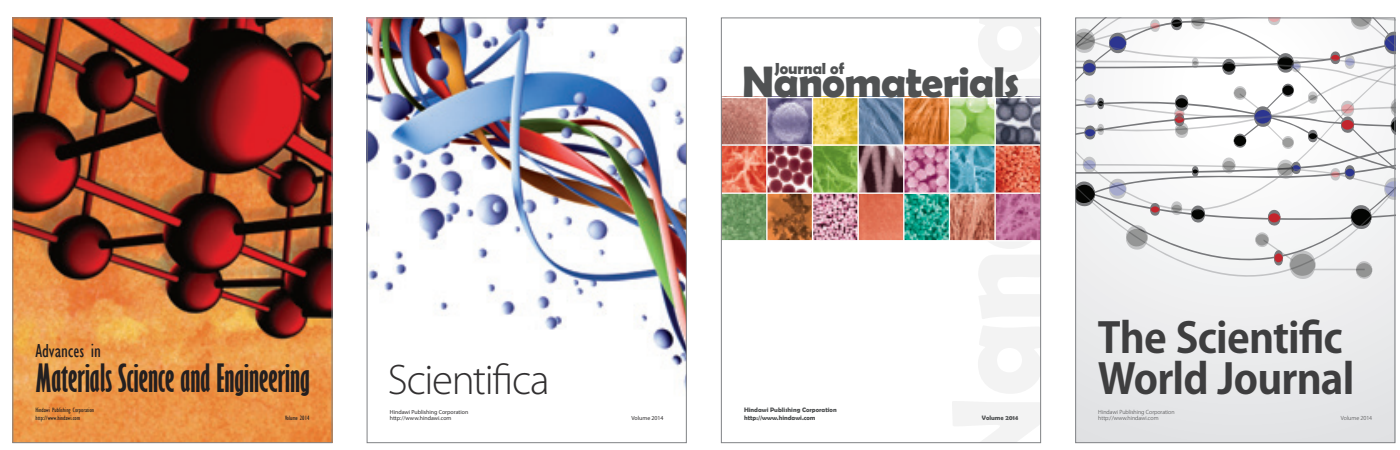

\section{The Scientific World Journal}
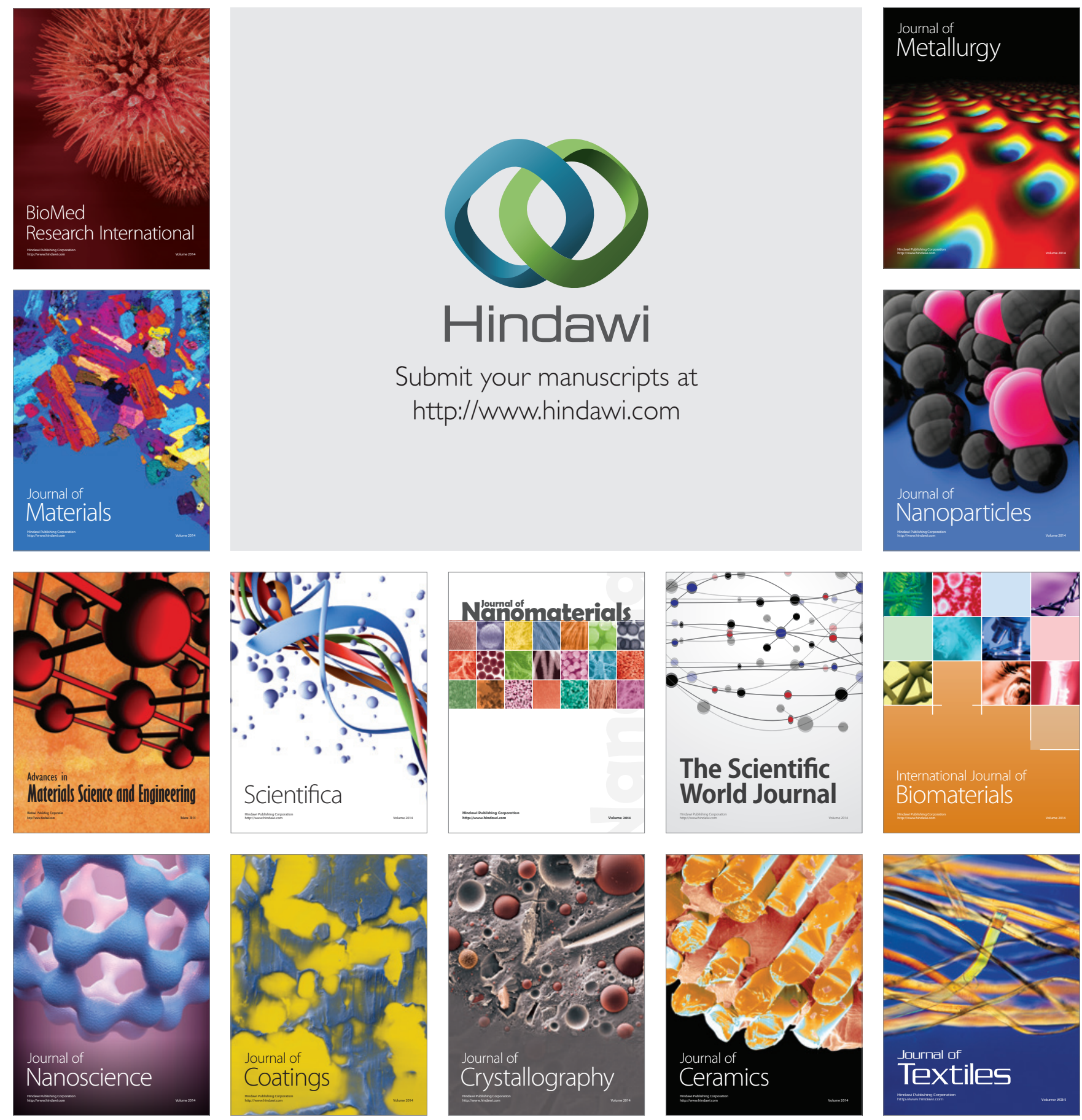